\title{
DETERMINING THE MINIMUM DATA SET FOR DIABETES REGISTRY
}

\section{Type of article: conference abstract}

Masoud Sotoudehfar1, Zahra Mazloum Khorasani2, Zahra Ebnehoseini3*, Kobra Etminani4, Mahmoud Tara5 and Robab bigom Aboutorabi6

1: Ph.D. Student Medical Informatics, Department of Medical Informatics, School of Medical, University of Medical Sciences, Mashhad, Iran.

2: Assistant Professor of Endocrinology, Endocrine Research center, Mashhad University of Medical Sciences, Mashhad, Iran.

3: Ph.D. Student Medical Informatics, Department of Medical Informatics, School of Medical, University of Medical Sciences, Mashhad, Iran.

4: Ph.D. in Health Information Management, Assistant Professor, Department of Medical Records and Health Information Technology, School of Paramedical Sciences, Mashhad University of Medical Sciences, Mashhad, Iran.

5: Ph.D. in Health Information Management, Assistant Professor, Department of Medical Records and Health Information Technology, School of Paramedical Sciences, Mashhad University of Medical Sciences, Mashhad, Iran.

6: Associate Professor of Endocrinology, Endocrine Research center, Mashhad University of Medical Sciences, Mashhad, Iran.

*Tel: +98.09370802011, E-mail: EbnehoseiniZ1@Mums.ac.ir

\begin{abstract}
Introduction: The number of people with diabetes's increasing. More than 220 million people have diabetes, more than $70 \%$ of whom live in middle and lower-income countries. already exist many innovations around the world on improving the managed care of diabetes .diabetes registries are one of them. in Iran, development and evaluation of diabetes information systems is one of the most research priorities. since defining health regulations and evaluation of diabetes prevention programs depend on the powerful information system, but in Iran don't exist complete information about incidence and prevalence of diabetes. determine standard data elements (Des) and design diabetes registry is one the most important country requirements. the main purpose of this study is investigating to this subject.

Methods: This is a descriptive- analytic study. Resource related to diabetes DEs collected from selective minimum data sets. Then diabetes DEs set derived from selective minimum data sets were investigated in focus group sessions with endocrine specialists, health informatics, and health information management. Duplicate DEs were removed and similar DEs were combined. Then seven endocrine specialists evaluated diabetes DEs set. They determine the value of each DEs using the Delphi technique (scores range from 0 to 5). The DEs that received more than $75 \%$ of grade 4 and 5 remained in the study. Following the expert opinion, the final version of the diabetes DEs set was designed.

Results: According to literature review 455 DEs included studying, after Delphi sessions, 293 data element remained to study. Main categories of DEs are:1-patient demographic characterizes (12 DEs), 2-patient referral (5 DEs), 3-diabetes care follow up (15 DEs), 4-physical exam, chief complaint and assessment (40 DEs), 5-history (such as: individual, grow up, family, drug abuse) (10 DEs), 6-pregnancy management (13 DEs), 7-screening (10 DEs), 8-specialty evolutions ( such as: cardiovascular (18 DEs), neuropathy (16 DEs), nephropathy (7 DEs), teeth and mouse (3 DEs), eyes (14 DEs), psychology situation (2 DEs), sexual ability (1 DEs)), 9-laboratory exams (33 DEs), 10-drugs (oral antidiabetics drugs (14 DEs), injectable antidiabetics (7 DEs), lipid (11 DEs), hypertension (20 DEs), anti placates (2 DEs)), cardiac (3 DEs), preparing insulin method (5 DEs)), 11-physical activity (4 DEs),12- diet (12 DEs), 13-education and self care (13 DEs).

Conclusion:In the study diabetes, DEs set were determined that provide appropriate yield for data gathering and record all required information for diabetes care. Hence diabetes is a chronic disease
\end{abstract}


Medical Technologies Journal, Volume: 1, Issue: 4, October-December 2017, Pages:76-136. Doi :

https://doi.org/10.26415/2572-004X-vol1iss4

and Patients suffer from it for years, implementation diabetes DEs can improve documentation and improve diabetes care.

KEYWORDS: Minimum Data Set, Diabetes mellitus, Diabetes

\section{Declaration of conflicts}

This abstract is selected from the First International Congress of Diseases and Health Outcomes Registry and First National Congress of Medical Informatics, 14-17 February 2017, Mashhad, Iran

\section{Authors' biography}

No biography.

\section{References}

No references. 